Stoa

Vol. 2, No. 3, 2011, pp. 75-92

ISSN : 2007-1868

DISTRIBUCIÓN DEL CONOCIMIENTO Y EXCLUSIÓN SOGIAL: EL PAPEL

DE LA UNIVERSIDAD EN LA SOCIEDAD DEL CONOCIMIENTO

Janette Alejandra González Hernández

RESUMEN: El siguiente es un ensayo crítico acerca del papel de la universidad como institución, misma que puede ayudar a abatir la exclusión social derivada del conocimiento tecnológico y científico en la sociedad del conocimiento. Propongo algunas líneas de cómo debe orientarse la institución de educación superior hacia un mejor proceso de distribución social del conocimiento. De lo que se trata es que la universidad se convierta en actor sustantivo de la distribución social, justa y equitativa, del conocimiento que ayudará a disminuir el rezago educativo. Las palabras clave son: exclusión social, conocimiento, ciencia y tecnología, Espacio Iberoamericano del Conocimiento y universidad.

PALABRAS CLAVE: exclusión social; conocimiento, ciencia y tecnología; Espacio Iberoamericano del Conocimiento y universidad; dostribución social del conocimiento.

SUMMARY: This paper is a critical essay about the role of the university as an institution which is able to support in declining social exclusion resulting from technological and scientific knowledge in the society of knowledge. I suggest some lines of how to orient the Institutions of Higher Education toward a better process of social distribution of knowledge. The challenge is that universities become mean actors of a social, fair and equitable distribution of knowledge in order to contribute to the reduction of educational gap.

KEY WORDS: social exclusion; knowledge, science and technology; Ibero-American Space of Knowledge and University; social distribution of knowledge.

\title{
Introducción
}

La exclusión social se define como el hecho donde "las personas y grupos son total o parcialmente excluidos de su completa participación en la sociedad 
en que viven" (Trouillot, en Figueroa 2000). Este proceso implica la no integración dentro de actividades políticas, culturales y económicas. De ahí que la exclusión social no sólo sea un tema del ámbito económico, sino también simbólico.

En nuestro país existen, desde hace algunos años, la exclusión social debida al conocimiento científico y tecnológico, que se suma a las ya existentes: a la exclusión económica, política, de género, cultural, entre otras.

La exclusión social en el ámbito del conocimiento genera una brecha que divide a los que pueden conocer y a los que no pueden, o mejor dicho, los que tienen acceso al conocimiento y los que no pueden tener acceso al conocimiento. Y no se trata de capacidades fisiológicas para el ejercicio de la inteligencia sino que el conocimiento científico y tecnológico exige mínimos básicos de saber a las personas donde va a ser depositado. Por ejemplo para utilizar una computadora ${ }^{1}$, hay que por lo menos, saber leer y escribir en español o tal vez un poco de inglés. Lo que deja fuera al cúmulo de personas que no saben leer ni escribir y que no hablan inglés, por ejemplo a los indígenas o campesinos, a los adultos mayores o a personas en condiciones de pobreza patrimonial que en México son más del 50 por ciento de la población. ${ }^{2}$ Eso en cuanto a la educación, pero por ejemplo para que la misma computadora opere es necesario que haya luz eléctrica, ahí ya se excluye a un buen número de personas que viven en el ámbito rural y que no pueden acceder a la energía eléctrica ya sea por un factor económico o por marginación y olvido de las instituciones.

\footnotetext{
${ }^{1}$ Figueroa, A., (2000), "La exclusión social como una teoría de la distribución" en Exclusión social y reducción de la pobreza en América Latina y el Caribe, Costa Rica, FLACSO/Banco Mundial. En este texto se pueden consultar diversas formas de exclusión social, entre ellas, las que viven campesinos e indígenas en América Latina. En este texto se incluye un trabajo sobre la adopción de la tecnología en 13 microrregiones del continente que muestra cómo los campesinos indígenas que no pueden acceder a las nuevas herramientas tecnológicas, los abonos o nuevas semillas, por no contar con requerimientos básicos de educación básica y bilingüe.

${ }^{2}$ Según datos del INEGI, en el conteo realizado en el 2005, México tenía una población total de 103 597366 personas. Por su parte Jonathan Heath (2007) muestra que en el 2005 el 47 por ciento de la población mexicana se clasificó como pobre, mientras que en pobreza extrema se ubicó el 18.2 por ciento de la población. Esto da como resultado que en aquel año el 65.2 por ciento vivía en condiciones de pobreza patrimonial. Así miso el presidente de la república, en el 2007 reconoció que el 50 por ciento de la población vive en una situación de pobreza patrimonial. Estos datos los dio a conocer a coordinadores parlamentarios al iniciar su sexenio en el 2007. (El Universal, jueves 17 de mayo, 2007)
} 
Según la encuesta del uso de las tecnologías ${ }^{3}$ realizada por el INEGI en el 2008, la televisión abierta es el tipo de tecnología a la que más tienen acceso los mexicanos, ya que el 93.2 por ciento de la población recibe la señal abierta en sus hogares, de un total de $103,263388^{4}$ de habitantes. En segundo lugar está el servicio telefónico, ya sea fijo o celular, en México el 75.5 por ciento de hogares tienen acceso a este tipo de tecnología. Por otro lado, a penas el 25.7 por ciento de hogares tienen una computadora en su casa y sólo el 13.5 por ciento de hogares tienen acceso al Internet. México está muy por debajo de países como Alemania, Suiza, Holanda o Corea donde más del $60 \%$ de hogares tienen conexión a Internet.

En nuestro presente paradójico, las tecnologías de la información no sólo acercan a la gente y acortan distancias como el teléfono, no sólo entretienen como la televisión o el Internet, también son un apoyo en la adquisición de nuevos conocimientos. Conocimientos que pueden cambiar la vida de las personas para mejorarlas en un sentido ideal.

En México, la televisión abierta es la tecnología que más se utiliza, sus contenidos son criticables y su uso obedece más al entretenimiento y de ello se sirven las grandes compañías para colocar productos en un espectro amplio de la población. Por otro lado el uso del teléfono, en la mayoría de los casos, es instrumental, sirve para conectar a una persona con otra, acortar distancias, eliminar costos, pero sus objetivos son efímeros e inmediatos. Finalmente el Internet integra los dos objetivos anteriores, el de entretener y comunicar, a uno nuevo que es la adquisición de información y datos que ponen las bases para la interiorización de conocimientos nuevos. De cualquier índole, no sólo académica. Una persona con conexión a Internet puede aprender desde nuevas técnicas de repostería hasta cómo construir grandes edificios. Es así pues que las tecnologías de la información, nos divierten, nos entretienen y nos ayudan a adquirir nuevos saberes. Lo deseable es que todas las personas tengan acceso a todas las tecnologías y a todos los conocimientos. Deberé matizar

\footnotetext{
${ }^{3}$ Hickman (2001) define a la tecnología como: a) el conjunto de técnicas, herramientas y artefactos, destrezas, incluso documentación b) los sistemas que exhiben y dependen de esas cosas c) la aplicación de la ciencia teórica y d) la investigación o incluso la investigación sistemática dentro de los elementos referidos.

${ }^{4}$ INEGI (2005) II Conteo de Población y Vivienda.
} 
que la información por sí misma no es conocimiento, la información es conocimiento cuando es valorada epistémicamente por un sujeto (Olivé, 2008).

Este texto mostrará desarrollará una propuesta de distribución social del conocimiento propia para la universidad. Orientada a que la institución potencialice sus capacidades para distribuir el conocimiento y dirija su atención fuera de las aulas y la educación formal, que llegue a personas que no tienen educación tradicional, entendida como los estudios de primaria, secundaria y preparatoria.

Antes de avanzar es importante decir que este texto es parte de la reflexión que realicé como resultado de mi trabajo de tesis de maestría en Filosofía de la Universidad Veracruzana, misma que llevó el título de: El conocimiento como moneda de progreso. El concepto de distribución social del conocimiento en la Universidad Veracruzana; un estudio de caso.

\section{La sociedad del conocimiento}

La exclusión social debida al conocimiento se da porque el conocimiento existente es acaparado por algunos pocos que gozan de los privilegios sociales, económicos y educativos y porque las instituciones que pudieran distribuir el conocimiento, no lo hacen o lo realizan de formas poco apropiadas y limitadas. El concepto de distribución social del conocimiento afirma que existe un bien intangible, el conocimiento, que debe ser distribuido. Este concepto supone que existen instancias donde se crea el conocimiento, se distribuye y se intercambia. Uno de estos espacios es la Universidad y en ella ahondaremos más adelante.

Incluso Olivé afirma que la distribución del conocimiento no se basa en criterios de justicia y no es un hecho democrático y abierto:

La forma distribuida de producción del conocimiento no significa una democratización del conocimiento, en el sentido de que sea público y accesible a todos, ni una disminución de la exclusión de millones de seres humanos de sus beneficios. Por el contrario, ni siquiera hay garantías de que quienes participan de una red se beneficien del conocimiento producido distribuidamente, o siquiera sepan cuál es realmente el producto final(Olivé 2004, p. 20).

La distribución social del conocimiento no es equitativa, ni justa, eso confirma que es un proceso que acontece en una sociedad liberal, capitalista. Donde existe un bien que se distribuye, según el mercado, entre las personas que 
puedan pagarlo o tienen el mínimo básico para utilizarlo. Éste es un acontecimiento que agudiza la diferencia de clases donde algunos pocos, tienen acceso al conocimiento científico y tecnológico que les puede ayudar a vivir bien. Y por el otro lado están los campesinos, los indígenas o las personas en situación de pobreza que viven en la ciudad que a falta de mejores oportunidades de empleo, se colocan en trabajos mal remunerados y no pueden aspirar a continuar la educación. Todos ellos, que son mayoría, quedan fuera de la idea de obtener nuevos conocimientos que los podrían llevar a vivir mejor, a mejorar sus condiciones de salud, de educación y accederían a nuevas formas de tecnología.

La distribución social del conocimiento pertenece a un campo semántico propio del presente conocido como sociedad del conocimiento. Este concepto refiere que la sociedad se sustenta en el conocimiento. El conocimiento es el principio legitimador de las actividades humanas y para autores como Bell (1973) o Krüger (2006) es el factor de cambio del presente. Gracias al conocimiento se han logrado transformaciones significativas en el ámbito de la salud, la educación o la guerra. Además la vida cotidiana ha cambiado radicalmente en las últimas décadas.

El conocimiento como hecho ontológico ha transformado la vida de los individuos. Pero no todos pueden estar dentro de esta realidad, hay quienes quedan al margen de esa transformación. Krüger considera que el conocimiento es un recurso económico, hasta puede entenderse como una mercancía, en ese sentido es un bien escaso y es necesario trabajar para obtenerlo. El conocimiento se concentra en universidades o institutos dejando rezagados a los que no pueden acceder a esos espacios.

Krüger también llama a este momento histórico, sociedad del desconocimiento, debido al cúmulo de conocimientos que día a día aparecen publicados, impresos o en la red, y que la gente no puede acceder a todos ellos. Entre más conocimiento hay, más se desconoce, lo cual genera formas de exclusión social. Bell preveía dos variables más, el ritmo y la magnitud: el primero se refiere a que el conocimiento se genera y se difunde en un ritmo acelerado eso permite que la magnitud de conocimiento sea mayor. Hay más conocimiento sobre el mundo y este se genera y se difunde exponencialmente. Debido a estos factores una persona no puede estar al tanto de todo el conocimiento, 
aunque sea un experto, mucho menos el individuo no iniciado. De ahí que desconozca más de lo que se conoce. Krüger matiza que no es que en el presente exista más conocimiento que en otro momento histórico, proporcionalmente hablando, sino que el cúmulo de conocimiento existente impacta más sobre la sociedad, incluso el aparato normativo queda subordinado al conocimiento, los gobiernos consultan primero al científico experto que al jurista por lo que ahora el conocimiento domina desde las bases administrativas del gobierno hasta las formas de nacer o morir. Por su parte Drücker (en Monstuschi, 1999) explica que anterior a este momento histórico el conocimiento se aplicaba a instrumentos, procesos y productos. Lo que dio como resultado la Revolución Industrial. Después y hasta la segunda guerra mundial el conocimiento se aplicó al trabajo y así se generó la Revolución de la Productividad. Finalmente en la Sociedad del Conocimiento se aplica el conocimiento al propio conocimiento para obtener una clase superior y más avanzada. Se trata de una búsqueda del conocimiento a partir del conocimiento para obtener más ganancias, una vida más cómoda, ser más bello, triunfar en la guerra, descifrar el genoma humano, entre otras cosas. La ciencia y la tecnología anclaron en el puerto de la sociedad del conocimiento para controlarlo todo, medirlo todo, llevarlo todo, administrar todo, opinar sobre todo. Son las herramientas fundamentales en la sociedad del conocimiento. Están en todos lados y a todos impactan. Conceden mejorar la calidad de vida, los hombres hacedores de ciencia y tecnología sintetizan medicinas para las enfermedades que atacan al cuerpo, construyen viviendas inteligentes, o acercan a familias y a amigos a través de las tecnologías de la información (internet, teléfono, televisión, por mencionar algunas); y al mismo tiempo ciencia y tecnología generan grandes debates éticos, por ejemplo: la biotecnología, con la alteración de productos naturales llamados transgénicos; también está la investigación genetista, con la polémica de la clonación y las células madre. Ciencia y tecnología son armas de doble filo que deben ser vistas a través del microscopio de la racionalidad y de la ética. Lo anterior tiene pertinencia en el presente, en lo que Bell (1973) llama sociedad post-industrial, que surgió luego de la segunda guerra mundial y se ha desarrollado con el incremento del conocimiento científico y tecnológico. Marx (2001) consideró que la producción era el momento más importante de la economía ya que era la única que podría generar valor, y es 
que el resto de momentos, el intercambio, la distribución y el consumo dependen de lo que se crea en esta etapa, en los otros momentos no se genera valor sino ganancia, entendida como plusvalía. Bell considera que en la sociedad post-industrial la economía se concentra en el sector terciario, el de los servicios y no ya, en la producción. Esto ha generado cambios importantes en la estructura social, por ejemplo: el surgimiento de una nueva élite conformada por aquellos que ofrecen servicios de tecnología, como los de telefonía móvil o internet, los ingenieros o los técnicos. Además esta sociedad post-industrial tiene una racionalidad orientada a fines que, como menciona Weber ${ }^{5}$, tiene anclaje en la burocracia, donde uno de los elementos principales es que los funcionarios burócratas dominen una tecnología de la administración, por ejemplo: la computadora, el fax, la copiadora, etc. Por su parte Bell sostiene que la sociedad post-industrial trae consigo un cambio en la estructura social y sus alcances son políticos y culturales. Por su puesto que dichos cambios impactan en la tradición, en la experiencia con la realidad, y el lenguaje es motor de dichos cambios. De forma general la sociedad post-industrial consiste en:

1. "La consolidación de la ciencia y los valores cognoscitivos como necesidad institucional básica de la sociedad. 2. La toma de decisiones cada vez más técnicas involucra a los científicos y economistas más directamente en los procesos políticos. a. La intensidad de las tendencias existentes hacia la burocratización del trabajo intelectual crea una serie de limitaciones a las definiciones tradicionales de los valores y empeños intelectuales. 3. La creación y la extensión de una intelligentzia técnica plantea problemas cruciales sobre la relación entre lo técnico y lo intelectual.” (Bell 1973, p. 64)

El autor destaca la importancia del conocimiento científico y tecnológico sobre cualquier otro tipo de conocimiento, además señala la ruptura de los valores tradicionales y el privilegio del conocimiento técnico sobre el intelectual.

\footnotetext{
${ }^{5}$ Weber, M., (1999), Economía y sociedad, México, Fondo de Cultura Económica. Este autor considera que las funciones de la burocracia moderna son: 1. Rige el principio de atribuciones oficiales fijas, ordenadas, por lo general, mediante reglas, leyes o disposiciones del reglamento administrativo es decir. 2. Rige el principio de jerarquia funcional y de la tramitación, es decir, un sistema firmemente organizado de mando y subordinación mutua de las autoridades mediante una inspección de las inferiores por las superiores. 3. La administración moderna se basa en documentos (expedientes) conservados en borradores y minutas, y en un cuerpo de empleados subalternos y de escribientes de toda clase. 4. La actividad burocrática especializada presupone un concienzudo aprendizaje profesional. 5. El desempeño de un cargo exige todo el rendimiento del funcionario. 6. El desempeño del cargo requiere del aprendizaje de normas y el dominio de una tecnología especial orientada a la administración, jurisprudencia, ciencias comerciales, entre otras. (ob cit.,1999: 717-719)
} 
Esta sociedad genera nuevos grupos sociales y la manifestación constante de grupos desaventajados que originan conflictos de poder y una distribución desigual de privilegios sociales. Esto quiere decir que la sociedad post-industrial tiene como eje al conocimiento pero el conocimiento no es para todos, se concentra en aquellos que lo generan y que reciben una retribución por ello, los científicos o, si no se es experto, el conocimiento permanece entre aquellos que tienen dinero para poseerlo o en quienes pueden tomar decisiones políticas o económicas.

La actitud hacia el conocimiento científico define al sistema de valores de una sociedad. La concepción medieval de la ciencia natural fue la de un "conocimiento prohibido". Los sacerdotes temían que el "conocimiento hiciese al hombre engreído> y que <recibiese algo de la serpiente". Durante los siglos cristianos, la naturaleza -en un sentido especial, claro está- había sido atribuida al orden satánico. La leyenda de Fausto, utilizada por Marlowe, da testimonio. Hacia el siglo XVII, la creencia en el poder expansivo del hombre ha comenzado a sustituir a la concepción anterior basada en el temor. En la Nueva Atlántida de Francis Bacon con la que éste no es ya el filósofo, sino el investigador científico. Y en la isla perecedera de Bensalem, la construcción más importante, la Casa de Salomón, no es una iglesia sino un centro de investigación (Bell 1973, p. 65)

El laboratorio o el centro de investigación se convierten en puntos sustantivos de la creación y consolidación del conocimiento. Éste ya no es un secreto, ya no guarda el velo mágico que tuvo en la edad media y en el renacimiento. Ahora es un conocimiento abierto pero con una trampa: está abierto sólo para aquellos iniciados y especialistas que puedan comprenderlo, replicarlo y socializarlo. Trabajar alrededor del conocimiento se ha vuelto una obsesión de algunos, la orientación es dominar el orden natural a través de la ciencia y la tecnología y controlar, de paso, la vida de las personas.

\section{Propuesta de distribución social del conocimiento}

La universidad debe contemplar algunos elementos básicos para lograr una distribución social del conocimiento justa y equitativa. A continuación desarrollaré cada una de las características que debe privilegiar la universidad a partir de una propuesta de distribución social del conocimiento.

¿Por qué una propuesta de distribución social del conocimiento? 
La universidad es un producto social en ella se concentran las virtudes o ausencias de la sociedad que la crea. Su definición es amplia y puede existir una diferente por cada orientación teórica. Para fines de simplificar diremos que existen dos grandes esquemas de sentido. El primero es el sentido positivo de la universidad, el cual destaca que la universidad funciona como institución social para el progreso y el bienestar, sintetizando las aspiraciones de la sociedad en la Modernidad. Mientras que el esquema de sentido negativo considera a la universidad como una institución para la reproducción de la dominación social, sintetizando los conflictos de clase propios de la modernidad (Ibarra Colado, 2001). En ese sentido la universidad educa a los individuos dentro de un esquema de control que los prepara para integrarse en el sistema laboral y económico, que no es otro que el capitalista. Para este trabajo es importante tomar en cuenta los dos esquemas de sentido pues considero que una de las obligaciones de la universidad es ayudar a generar progreso y bienestar, pero también creo que en sus prácticas recrea procesos de dominación y exclusión. Así mismo, la universidad es compleja puede entenderse como una organización social, por su estructura, y al mismo tiempo, como una institución debido a sus prácticas; por organización entenderemos el espacio en los que tienen lugar relaciones materiales y simbólicas entre agentes sociales diversos, ubicados en una estructura institucional definida (Op. Cit.). Así mismo es una institución debido a que las prácticas que en ella se realizan crean formas de relación social, valores, procesos culturales propios, así como normas, relaciones de control y de poder (Chavoya 2002, p. 57). Por su parte Berger y Luckman (1992) consideran que entender a la universidad como institución implica analizarla en su historicidad y en sus procesos de control ya que las instituciones tienden a controlar el comportamiento humano estableciendo pautas definidas de antemano que lo canalizan hacia una dirección determinada. Esta complejidad es lo que facilita y al mismo tiempo entorpece que las universidades asuman nuevas propuestas o líneas de trabajo. Es el caso de la distribución social del conocimiento. Cada vez se rompe más con la idea de que la vocación universitaria no es sólo la educación sino que la orientación es integrarse al contexto que la rodea, aportando sus conocimientos para fomentar el desarrollo de una colonia, una comunidad o una región. De lo que se trata es de abrir a la universidad a nuevas formas de entender la distribución social del conocimiento que vayan más allá 
de la educación formal (estudios de primaria, secundaria y preparatoria). Lo anterior tomando en cuenta que existen un grupo de personas históricamente excluidas, quienes por no tener educación formal, tener alguna discapacidad o vivir lejos de las instalaciones universitarias, no han podido acceder al proceso de apropiación social del conocimiento que en las universidades se genera. Cabe mencionar que en el país ya existen iniciativas de distribución del conocimiento específicas para disminuir exclusión social de personas pertenecientes a etnias indígenas, tal es el caso de la Universidad Veracruzana Intercultural, la Universidad Intercultural de Tabasco, la Universidad Intercultural del Estado de México o la Universidad Intercultural del Estado de Chiapas. Para estas universidades cuyo modelo educativo está orientado a formar profesionales desde la diversidad cultural existen programas especiales en línea para distribuir el conocimiento por medio de las tecnologías de la información. De la misma forma las universidades realizan actividades de extensión universitaria y culturales donde también se distribuye el conocimiento.

Aunque ya hay trabajo avanzado en el proceso de distribución social del conocimiento que realizan las universidades en México todavía es limitado y hace falta ampliar el esquema o complementar el que ya existe. El énfasis en mi trabajo está orientado a eliminar la exclusión social debida al conocimiento, sostengo que las universidades tienen que ampliar sus métodos de distribución social del conocimiento, de lo que se trata es de consolidar una universidad abierta, plural e incluyente. Mi propuesta se orienta a distribuir el conocimiento entre un grupo de personas cuyas características no tienen que ver con elementos de multiculturalidad ni con capacidades diferentes privativamente sino que se abre a otro grupo de individuos, históricamente excluidos, quienes no han tenido acceso al conocimiento que la universidad genera o posee. Intento reforzar la distribución social del conocimiento entre el sujeto excluido de la educación formal, quien no cuenta con los niveles básicos de educación, por ejemplo, no sabe leer o escribir. No maneja una segunda lengua, el español en caso de hablantes indígenas o el inglés en hablantes de español. No tiene conocimientos básicos de computación. Pero que realiza un oficio para obtener ingresos económicos que le garantizan el sustento. Como lo mencioné mi propuesta no excluye a personas de otras etnias indígenas o con capacidades diferentes, tampoco a campesinos y a personas del medio 
urbano, mucho menos a miembros de empresas o a dependencias gubernamentales

\section{3. ¿Qué conocimiento se va a distribuir?}

En primer lugar el conocimiento a distribuir es conocimiento pertinente al entorno. La Universidad tiene la responsabilidad de distribuir el conocimiento en todos los lugares en los que ya tiene presencia e incrementar los espacios de impacto debido a que la demanda de conocimiento es amplia. La Universidad debe distribuir todo tipo de conocimiento, que las personas puedan acceder al tipo de conocimiento que les beneficie, esto requiere distribuir todas las áreas del conocimiento, desde las ciencias básicas como las matemáticas o la física hasta las ciencias aplicadas como la agronomía, la contaduría o el derecho. Se debe distribuir un conocimiento que fomente el desarrollo regional, que pueda impactar en cualquier sujeto social que lo desee ya sea una persona o una microempresa. El conocimiento que la universidad distribuya debe estar mediado por la racionalidad Mosterín considera que la racionalidad científica es un cúmulo de creencias sometidas a revisión constante. Así que ámbitos que podrían no parecer racionales como la cultura o la tradición, se vuelven racionales al momento de someter las creencias a procesos de revisión y autocrítica. La racionalidad implica cambio y aceptar una serie de errores que de la propia vida o ideas van surgiendo ${ }^{6}$, no sólo orientada a fines sino una racionalidad sustentada en el bienestar de los que habitan en el presente y sobre todo de los que estarán en el futuro. Además la distribución socia del conocimiento debe tener un fuerte anclaje ético que permita asegurar el porvenir de la sociedad.

La propuesta de distribución social del conocimiento que planteo es la siguiente:

-Derecho Universal al conocimiento. La Universidad debe tener en cuenta que todas las personas tienen derecho a la generación y disfrute del conocimiento. De ahí que todos tengan derecho a la educación, al acceso a la ciencia y la tecnología así como a participar de las actividades culturales y recreativas. Con todos, me refiero tanto a alumnos de la institución como a habitantes del lugar donde está inserta la universidad, que no necesariamente han reci-

${ }^{6}$ Mosterin, J., (1978), Racionalidad y acción humana, Madrid, Alianza Editorial. 
bido una educación básica formal, esto obligaría a la universidad a integrar en su población objetivo, a éste nuevo grupo de personas, antes excluido, para proveerles de conocimiento que pueda mejorar su desempeño laboral e incrementar sus capacidades intelectuales.

-Distribución social del conocimiento democrática.

Si todos tienen derecho al conocimiento, todos tienen el derecho de tomar decisiones que impacten sobre la construcción social del conocimiento. Esto es que la universidad debe consolidar mecanismos de tomas de decisiones que incluyan a un mayor número de personas, siempre tomando en cuenta a los habitantes de las comunidades o localidades donde trabaja la institución. De esta manera la universidad debe ser agente de cambio social junto con las personas a las que beneficia. Para distribuir democráticamente el conocimiento las universidades deben reforzar la formación de expertos en la divulgación. $\mathrm{Al}$ respecto Olivé considera que es necesario:

formar especialistas en el estudio del sistema de ciencia y tecnología y sus relaciones con la sociedad, capaces de analizar y promover las prácticas y redes institucionales y sociales que fomentan los nuevos modos de producción, distribución y aplicación del conocimiento, atendiendo a sus dimensiones cognitivas, éticas, axiológicas, sociales, educativas, económicas, políticas, jurídicas, culturales y ambientales (Olivé 2008, p. 43)

Se trata de llevar el conocimiento de una forma fácil y entretenida a todos los estratos sociales. El experto en divulgación del conocimiento no debe ser el que más sepa sino el que promueva y genere el diálogo de saberes. Con esto me refiero a que la distribución social del conocimiento no sólo debe ser vertical, donde la institución de a conocer el conocimiento a la sociedad, sino que la sociedad pueda compartir su conocimiento cultural y tradicional a la institución y éste sea tomado en cuenta.

-Inclusión Social. La universidad debe contribuir a eliminar la exclusión social que genera el conocimiento estableciendo mecanismos y realizando acciones que tiendan a disminuir la exclusión social en sectores marginados de la región donde se inserta, tanto en el ámbito rural, como en el ámbito urbano, así mismo que incluya dentro de su población objetivo, a las personas que no gozan con los mínimos establecidos para recibir una educación formal. Poniendo a su disposición una plataforma presencial y/o virtual que acerque el conocimiento a las comunidades y personas que lo necesiten. Esto facilitará 
la incorporación de cultura tecnocientífica a personas que antes vivían al margen de la misma, ya sea por situaciones económicas o sociales. La apertura de de la universidad hacia la sociedad que no ha tenido educación formal se puede generar ofreciendo talleres que faciliten la especialización de las personas en algún ámbito del conocimiento que sirva para complementar y mejorar el oficio que las personas ya desempeñan.

Todo esto debe llevar a consolidar la relación de la universidad con su entorno para constituirse como agente de cambio social que ayude a mejorar la vida de las personas. Esto se traduce en incrementar la vinculación de la universidad con las empresas. No sólo las micro o grandes empresas, la institución deberá relacionarse con micro y pequeñas empresas que soliciten el apoyo para la transferencia de conocimiento, capacitación que fortalezcan el desarrollo local y regional.

Algunas universidades han dado pasos en la inclusión de personas que pertenecen a etnias indígenas, a través de la Universidad Intercultural, facilitándoles una educación formal a nivel licenciatura o maestría o cursos focalizados. Lo que hace falta es incorporar a personas con capacidades diferentes en las actividades educativas, de extensión y fomento de la cultura. El primer paso será crear la infraestructura básica, como rampas y espacios destinados a personas con discapacidades motrices o visuales, facilitando textos en lenguaje braile o programas de computación especializados.

- Consolidación de sistemas científicos-tecnológicos. Los sistemas científicostecnológicos contemplan la unión de las universidades, el estado y la sociedad civil para realizar acciones conjuntas en materia de creación, distribución y aplicación del conocimiento. No sólo se pretende que universidades, instituciones de gobierno y la sociedad se involucren en el proceso de creación de conocimiento sino que se de cabida al debate público de las controversias científicas y tecnológicas. ${ }^{7}$ Aún cuando en las universidades del país ya realiza actividades de vinculación con las instituciones de gobierno y empresas privadas. La distribución social del conocimiento justa y equitativa requiere

${ }^{7}$ Broncano (2000) considera urgente que los debates tecnológicos se lleven a la discusión pública y no se queden sólo en las charlas de los grupos de expertos o de beneficiarios directos. Yes que, como ya lo ha mencionado, las decisiones tecnológicas afectan a las generaciones actuales y también a las futuras, lo que supone una responsabilidad moral. Los debates podrían girar alrededor de la construcción de una presa en alguna región o de la conformación una planta industrial, las discusiones deben ser públicas e incluir el mayor número de ideas. 
incrementar el lazo entre las partes fortaleciendo la relación entre todos los actores involucrados: científicos, funcionarios universitarios, empresas, instituciones del estado así como representantes de la sociedad civil. Estos sistemas científicos son incluyentes y ayudan a fortalecer la distribución social del conocimiento ya que son espacios plurales de creación de saberes.

Los sistemas científicos tienen un estructura de normas y de valores que suele variar de unos a otros. Si bien la producción de conocimiento es común a todos ellos, y en todos existen controles de calidad epistémicos, ni siquiera éstos son los mismos en cada caso (...) Estos sistemas científicos-tecnológicos obedecen a una amplia variedad de intereses y de valores (...) en estos sistemas entra en juego una variedad de valores: epistémicos, técnicos, éticos, económicos, jurídicos, ecológicos, sociales, militares, religiosos, estéticos, políticos aunque no siempre estén presentes todos ellos (Olivé 2008, pp. 22-23)

La universidad junto con las dependencias estatales y municipales deben trabajar en conjunto para consolidar la creación del sistema científico y tecnológico y así puedan compartir los valores técnicos, éticos, jurídicos, ecológicos, y sociales. Esto beneficiaría a ambas partes, por un lado la universidad se consolidaría como agente de cambio en la región al compartir su conocimiento y sus avances científicos y tecnológicos con su entorno., además mejoraría su status social y ampliaría su impacto. Por su parte las instituciones estatales se beneficiarían con los conocimientos que genera la universidad. Además de generar plataformas donde se puedan discutir y resolver problemas de índole técnica, tecnológica, jurídica, ética y social.

- Pluralidad en la distribución del conocimiento. La creación de sistemas científicos-tecnológicos que compartan objetivos, motivaciones y criterios de éticos conlleva la aceptación de una realidad plural que pueda generar un Estado plural ${ }^{8}$

${ }^{8}$ Olive considera que la idea fundamental de un Estado plural en un país multicultural es la de un Estado que no está al servicio de ningún pueblo ni cultura en particular, y que es capaz de articular un mínimo de intereses y valores comunes, al que legítimamente se adhieran todos los pueblos y sectores que participan en el desarrollo del proyecto nacional, aunque cada uno de ellos tenga sus propias razones para hacerlo. Lo importante es que todos reconozcan la legitimidad de las instituciones estatales y colaboren en la construcción y realización de ese proyecto. ${ }^{9}$ donde la opinión de un actor social tiene el mismo el mismo peso del otro actor social dentro del sistema científico. Aún cuando los intereses de los actores son diversos, la opinión de todos debe ser escuchada y debatida.

Por otro lado la pluralidad no sólo compete al orden del sistema científico y tecnológico también debe estar inserta dentro del discurso universitario como uno de los valores más importantes, mismo que 
Aún existe la creencia de que el conocimiento es un elemento con poderes metafísicos que puede cambiar la sociedad y que por lo mismo sólo debe estar al resguardo de algunos iniciados. Esta iniciativa contempla bajar del pedestal al conocimiento científico y tecnológico que generan las universidades, esto significa que:

El significado práctico de estos resultados en el ámbito educativo involucra entonces, además de una drástica renovación de los contenidos educativos, dos importantes cambios actitudinales y metodológicos: el abandono del papel del profesor como meta-experto o como mediador autorizado y privilegiado del conocimiento experto, por un lado, y la promoción de la participación crítica y creativa de los estudiantes en la organización y desarrollo de la docencia, por el otro lado(López y Luján 1996, p. 277)

El papel del experto ha jugado un papel importante en la sociedad, sobre todo en esta sociedad del conocimiento, no obstante, es necesario equilibrar el uso del conocimiento. Más personas deben tener acceso a saberes antes destinados a los iniciados en un tema y los expertos deben poner a disposición de las personas su cúmulo de conocimiento. Sólo así se podrá consolidar una distribución social del conocimiento justa y equitativa.

\section{Conclusiones}

La sociedad del conocimiento todavía está lejos de consolidarse en una realidad como la mexicana. El cambio social, en nuestro país, se explica por diferentes factores que no necesariamente tienen que ver con el conocimiento. Existen profundas desigualdades que se arrastran históricamente. Por lo que tener conocimiento sólo es una forma más de agravar la exclusión social. Además quedan preguntas por resolver como: ¿qué tipo de conocimiento necesita un país como el nuestro para consolidar una sociedad conocimiento? ¿Este país necesita conocimiento para lograr un cambio social que beneficie a todos o sólo se trata de un cambio de actitud o cultural? ¿Tiene vigencia la sociedad del conocimiento en una realidad así o sólo nos conformaremos con ser países

permite que exista una diversidad de opiniones que son tomadas en cuenta para la toma de decisiones dentro de la universidad. El pluralismo también implica conceder una participación más activa de la sociedad civil en la universidad, misma que fortalecería el vínculo de la con su entorno. Se trata de romper con la idea de que la universidad es una institución monolítica alejada de la sociedad, lo óptimo sería incluir las opiniones de las personas que viven en el entorno inmediato de la universidad. -Desmitificación del conocimiento científico y tecnológico. 
al margen del cambio que se genera en otros lugares del orbe? Vivimos en un contexto de crisis internacional que nos ha alcanzado a todos. Si no hiciéramos nada y continuáramos sobre una línea pesimista, lo único que quedaría es esperar y ver cómo se incrementa la exclusión social y la marginación. La sociedad del conocimiento augura un futuro con mejor calidad de vida pero sólo para algunos privilegiados que tienen el acceso a las tecnologías y al conocimiento. Por eso es necesario mover nuestros recursos para consolidar un espacio de justicia donde todos puedan acceder al conocimiento y la información y así mejorar su vida. No se trata de reflexionar alrededor de buenas intenciones y señalar lo que es bueno y lo que no lo es. Se trata de actuar, el presente nos obliga a pensar pero también a realizar acciones concretas para mejorar nuestra vida y la vida de las personas que nos rodean, para que el conocimiento llegue a más personas, para que se reduzcan los niveles de exclusión social.

La universidad no debe hacer a un lado su vocación de generar conocimiento y educar, pero más que nunca deben fortaleces la investigación y el desarrollo. Deben ser motores de la distribución social del conocimiento justa y equitativa tomando en cuenta los criterios, de universalidad, democracia, pluralidad, desmitificación del conocimiento e inclusión.

El reto que se nos presenta es transdisciplinar, no compete sólo a los filósofos pensar en lo que sería mejor para nuestra región; tampoco está en manos de los sociólogos operar programas que distribuyan mejor los recursos; ya no es sólo responsabilidad del ingeniero crear máquinas para mejorar la vida de la gente; ni los médicos son los únicos encargados de hacer sentir saludables a las personas. Ahora el reto es de todos. Sólo con la cooperación de saberes diversos será posible combatir reducir los niveles de exclusión social que vivimos. Será posible hacer llegar el conocimiento a más personas y mejorar la calidad de vida.

\section{Referencias}

Bell, D., 1973, El advenimiento de la Sociedad Post industrial, Alianza Universidad, Madrid. Berger, P. L. y Luckman, T., 1992, La construcción social de la realidad, Amorrortu, Buenos Aires.

Broncano, F., 2000, Mundos artificiales, Paidos, México. 
Chavoya Peña, M. L., 2002, La institucionalización de la investigación en la Universidad de Guadalajara, Universidad de Guadalajara, Guadalajara.

Ibarra Colado, E., 2001, La universidad en México hoy: gubernamentalidad y modernización, Universidad Nacional Autónoma de México/ Universidad Autónoma Metropolitana, México.

Castro, E. y Vega Jurado, J., 2009, "Las relaciones universidad entorno socioeconómico en el Espacio Iberoamericano del Conocimiento", Revista Iberoamericana de Ciencia, tecnología y Sociedad, $\mathrm{n}^{\circ} 12$ vol. 4,

http://www.oei.es/cienciayuniversidad/spip.php?article483.

Recuperado el 20 de enero del 2010.

Figueroa, A., 2000, "La exclusión social como una teoría de la distribución”, en Exclusión social y reducción de la pobreza en América Latina y el Caribe, FLACSO/Banco Mundial, Costa Rica.

Hickman, L., 2001, Philosophical Tools for Technological Culture. Putting Pragmatism to Work, Indiana University Press, Bloomington and Indianápolis.

INEGI, 2008, "Encuesta nacional sobre disponibilidad y uso de tecnología de información en hogares",

http:/ / www.inegi.org.mx/inegi/default.aspx?c=10660\&s=est.

Recuperado el 12 de enero del 2010.

Krüger, K., 2006, "El concepto de sociedad del conocimiento", Revista bibliográfica de geografía y ciencias sociales, Vol. XI, nº 683, Barcelona.

López Cerezo, J. y et al., 1996, Ciencia, tecnología y sociedad, Tecnos, Madrid.

Marx, K., 2001, El capital. Crítica de la economía política, Fondo de Cultura Económico, México.

Montuschi, Luisa, 1999, "Datos, información y conocimiento. De la sociedad de la información a la sociedad del conocimiento",

http://cema.edu.ar/publicacion/download/documentos/192.pdf.

Recuperado el 12 de enero del 2010.

Mosterin, J., 1978, Racionalidad y acción humana, Alianza Editorial, Madrid.

Olivé, L., 2008, La ciencia y la tecnología en la sociedad del conocimiento, Fondo de Cultura Económica, México.

2005, "La cultura científica y tecnológica en el tránsito a la sociedad del conocimiento", Revista de la Educación Superior, vol. XXXIV, 4, no.136. Octubre-diciembre, ANUIES, México.

Tiana Ferrer, A., 2009, "El Espacio Iberoamericano del Conocimiento: retos y propuestas",

http:/ / www.fundacioncarolina.es/esES/nombrespropios/Documents/

NPTiana0907.pdf.

Recuperado el 09 de diciembre del 2009. 
Toscano, J. C., 2009, "Espacio Iberoamericano del Conocimiento", Revista CTS, n 12 , vol. 4, pp. 85-87,

http://www.oei.es/cienciayuniversidad/spip.php?article483.

Recuperado el 10 de diciembre del 2009.

Recibido el 2 de Febrero de 2011

Aceptado el 15 de Febrero de 2011 